3. Yangco BG, Buchacz K, Baker R, Palella FJ, Armon C, Brooks JT, and the HIV Outpatient Study Investigators. Is primary Mycobacterium avium complex prophylaxis necessary in patients with CD4 $<50$ cells $/ \mathrm{mL}$ who are virologically suppressed on cART? AIDS Patient Care STDS. 2014;28:280-283.

4. Ticona-Huaroto C, Astocondor-Salazar L, MontenegroIdrogo J, Valencia-Mesias, Soria J. Infección por el complejo Mycobacterium avium-intracellulare en pacientes con VIH/ SIDA en un hospital peruano: una serie de casos. Rev Peru Med Exp Salud Publica. 2017;34(2):323-327.

5. Norma Técnica de Salud No 097-MINSA/DGSP-V.02. Norma Técnica de Salud de Atención Integral del Adulto con Infección por el Virus de la Inmunodeficiencia Humana (VIH) [Internet]. Lima: Ministerio de Salud; 2014. [Citado el 17 de diciembre de 2017]. Disponible en: http://www.digemid.minsa.gob.pe/ UpLoad/UpLoaded/PDF/Normatividad/2014/RM_9622014.pdf

7. Gopinath K, Singh S. Non-tuberculosis mycobacteria in TBendemic countries:are we neglecting the danger? PLoS Negl Trop Dis. 2010;4(4):e615. doi:10.1371/journal.pntd.0000615.

Correspondencia: Julio Maquera Afaray.

Dirección: Prolongación San Camilo 1022, Urbanización Vigil, Tacna, Perú. Correo electrónico:maquera.afaray.julio@gmail.com.

\section{ACTIVIDAD ANTIBACTERIANA FRENTE A Streptococcus mutans DE ACEITES ESENCIALES DE CINCO PLANTAS ALTO ANDINAS}

\section{ANTIBACTERIAL ACTIVITY OF FIVE HIGH- ANDEAN PLANT ESSENTIAL OILS AGAINST Streptococcus mutans}

\author{
Elsa Gladys Aguilar-Ancori',2,a, \\ Katya Valentina Aguilar-Ancori ${ }^{3, b}$, Bryan Garay ${ }^{3, b}$, \\ Vladimir Mamani ${ }^{3, \mathrm{~b}}$, Mercedes Maritza Quispe-Flórez ${ }^{1,2, \mathrm{c}}$.
}

Sr. Editor. El efecto antibacteriano de los aceites esenciales (AE) de plantas contra la bacteria cariogénica como Streptococcus mutans, se ha estudiado ampliamente ${ }^{(1-5)} \mathrm{y}$ algunos componentes han sido probados en formulaciones

\footnotetext{
Escuela Profesional de Biología, Universidad Nacional de San Antonio Abad del Cusco. Cusco, Perú.

2 Instituto Universitario de Enfermedades Tropicales y Biomedicina del Cusco. Cusco, Perú.

3 Escuela de Estomatología, Universidad Andina del Cusco. Cusco, Perú.

a Bióloga, magister en salud pública; ${ }^{\mathrm{b}}$ odontólogo; ${ }^{\mathrm{c}}$ bióloga.

Recibido: 18/10/2017 Aprobado: 22/11/2017 En línea: 05/04/2018
}

Citar como: Aguilar-Ancori EG, Aguilar-Ancori KV, Garay B, Mamani V, Quispe-Flórez MM. Actividad antibacteriana frente a Streptococcus mutans de aceites esenciales de cinco plantas alto andinas. Rev Peru Med Exp Salud Publica. 2018;35(1): 161-3.doi: 10.17843/rpmesp.2018.351.3610. de enjuagues bucales, pasta dental o geles, cuyos resultados mostraron una reducción del $S$. mutans en la saliva en formulaciones altamente concentradas. Estos hallazgos demuestran que dichas formulaciones aún no son suficientemente efectivas para mantener la higiene oral y prevenir la placa dental, en comparación con formulaciones de higiene oral de venta libre, cuya mezcla de sus componentes timol, eucaliptol y salicilato de metilo, mantienen la higiene oral y son más efectivos contra el S. mutans.

En la búsqueda de nuevos agentes anticariogénicos de origen natural, los AE de plantas alto andinas (Cymbopogon citratus, Piper elongatum, Minthostachys setosa, Schinus molle y Luma chequen) utilizadas en la medicina tradicional, han mostrado actividad antibacteriana sobre el S. mutans (1-5); por consiguiente, conocer su espectro químico y eficacia antibacteriana puede ser de utilidad para sintetizar productos anticariogénicos y generar nuevas formulaciones dentales más eficaces. El objetivo de esta comunicación es dar a conocer la actividad antibacteriana de cinco AE de plantas nativas alto andinas de Perú frente a S. mutans.

Aceites esenciales de $C$. citratus, $P$. elongatum, $S$. molle, $M$. setosa, $y L$. chequen comercializados por el Laboratorio Pukllay (Asociación Pukllasunchis, productores comerciales de $A E$ de plantas, Cusco, Perú) se utilizaron en el estudio. Su composición química se determinó a partir de $100 \mu \mathrm{L}$ de $\mathrm{AE}$ en un equipo de cromatografía de gases (Agilent $6890 \mathrm{~N}$ ) calibrado con una columna HP-5MS 5\% Fenil Metil Siloxane. Una cepa de S. mutans (ATCC 35668) que se cultivó en agar sangre $(\mathrm{BHI}+5 \%$ sangre de cordero) y caldo $\mathrm{BHI}$ a 37 ${ }^{\circ} \mathrm{C}$ por 24 horas fue utilizado en los ensayos. La actividad antibacteriana se determinó por el método del disco difusión en agar ${ }^{(5)}$. Los AE se disolvieron al 2,$5 ; 5 ; 7,5 ; 10 ; 15 ; 20$; 25; 30; 40; 50; 75 y $100 \%$ en Tween 20 . Una suspensión del inóculo de $S$. mutans fue diluido en $10^{-3} \mathrm{ml}$ de solución salina estéril ( $1 \mathrm{ml}$ de $10^{8}$ células $\left./ \mathrm{ml}\right)$ y se inoculó en placas Petri con agar Mueller-Hinton con $5 \%$ de sangre. Discos de papel de filtro de $6 \mathrm{~mm}$ de diámetro se empaparon en 15 $\mu \mathrm{L}$ de cada $\mathrm{AE}$ diluido y se colocaron sobre la superficie de las placas inoculadas. Como control positivo y negativo se utilizaron discos empapados con clorhexidina al $0,12 \%$ y Tween 20 , respectivamente. Las placas fueron incubadas en cámaras de anaerobiosis a $37^{\circ} \mathrm{C}$ durante 48 horas. Luego se realizaron las lecturas de los diámetros de los halos de inhibición en milímetros $(\mathrm{mm})$. Todos los ensayos se realizaron por triplicado.

El análisis cromatográfico reveló diez componentes químicos por cada AE. Citral (40,2\%), a-phellandrene (23,8\%), eucalyptol $(17,3 \%)$, a-pinene $(51,1 \%)$ y menthone $(21,9 \%)$ fueron los componentes predominantes de los AE de $C$. citratus, $S$. molle, $P$. elongatum, $L$. chequen y $M$. setosa, respectivamente (Tabla 1a).

La actividad antibacteriana de los $\mathrm{AE}$ sobre el $S$. mutans se muestra en la Tabla $1 b$. Los $A E$ de $C$. citratus y $P$. elongatum mostraron una potente actividad antibacteriana a partir 
Tabla 1. (a) Composición de los aceites esenciales S. molle, P. elongatum, L. chequen, M. setosa y C. citratus. (b) Actividad antibacteriana de los $\mathrm{AE}$ de $C$. citratus, $P$. elongatum $M$. setosa y $S$. molle sobre $S$. mutans (concentraciones de los AE vs. los halos de inhibición del S. mutans)

\begin{tabular}{|c|c|c|c|c|c|c|c|c|c|}
\hline \multirow{2}{*}{\multicolumn{2}{|c|}{ (a) Schinus molle }} & \multicolumn{8}{|c|}{ Composición química de los aceites esenciales } \\
\hline & & \multicolumn{2}{|c|}{ Piper elongatum } & \multicolumn{2}{|c|}{ Luma chequen } & \multicolumn{2}{|c|}{ Minthostachys setosa } & \multicolumn{2}{|c|}{ Cymbopogon citratus } \\
\hline Componente & (\%) & Componente & (\%) & Componente & (\%) & Componente & (\%) & Componente & $(\%)$ \\
\hline a-phellandrene & 23,8 & Eucalyptol & 17,3 & a-Pinene & 51,1 & Menthone & 21,9 & Citral & 40,2 \\
\hline Camphene & 12,6 & $y$-Terpinene & 12,1 & Eucalyptol & 10,1 & Carvacrol & 4,9 & $\beta$-Citral & 32,8 \\
\hline$\beta$-Pinene & 12,6 & a-Pinene & 4,4 & $\beta$-Pinene & 8,6 & Linalol & 2,9 & Myrcene & 9,1 \\
\hline a-Pinene & 11,8 & $\beta$-Pinene & 4,3 & Linalol & 7,3 & $\beta$-Caryophyllene & 2,8 & Geraniol & 4,4 \\
\hline Myrcene & 5,1 & $\beta$-Caryophyllene & 3,0 & Guaiyl acetate & 4,3 & p-Cymene & 1,4 & 6-Methyl-5-hepten-2-one & 3,7 \\
\hline Limonene & 3,8 & a-phellandrene & 2,2 & Valencene & 4,2 & Limonene & 1,0 & Linalol & 1,9 \\
\hline $\mathrm{y}$-Terpinene & 2,6 & a-terpinene & 1,5 & Limonene & 2,7 & a-Pinene & 0,6 & Geranyl acetate & 1,2 \\
\hline$\beta$-Caryophyllene & 2,0 & Limonene & 1,4 & $\beta$-Caryophyllene & 1,6 & $\beta$-Pinene & 0,6 & Undecanone & 0,9 \\
\hline p-Cymene & 2,0 & a-Terpineol & 1,3 & a-Terpineol & 1,3 & Thymol & 0,5 & Citronellol & 0,8 \\
\hline sabinene & 1,0 & Myrcene & 1,3 & $\begin{array}{l}\text { Isobutyl } \\
\text { isobutyrate }\end{array}$ & 1,0 & Myrcene & 0,4 & 2-Tridecanone & 0,7 \\
\hline
\end{tabular}

\begin{tabular}{|c|c|c|c|c|}
\hline \multirow{2}{*}{$\begin{array}{l}\text { (b) } \\
\text { Concentración (\%) }\end{array}$} & \multicolumn{4}{|c|}{ Halos de inhibición de los aceites esenciales sobre el $S$, mutans $(\mathrm{mm})$} \\
\hline & Schinus molle & Piper elongatum & Minthostachys setosa & Cymbopogon citratus \\
\hline 15 & - & - & - & 15,7 \\
\hline 20 & - & 10 & - & 17,7 \\
\hline 25 & - & 11,7 & - & 20,0 \\
\hline 30 & - & 14 & - & 20,7 \\
\hline 40 & - & 15 & 7 & 23,3 \\
\hline 50 & - & 17,8 & 8,8 & 32,7 \\
\hline 75 & 14,7 & 21,7 & 9,9 & 39,7 \\
\hline 100 & 16,3 & 25 & 11,3 & 50,3 \\
\hline
\end{tabular}

del 15 y $20 \%$ de concentración, respectivamente, cuyos diámetros de los halos de inhibición fueron 15,67 y $10 \mathrm{~mm}$, respectivamente. En contraste, los AE de $M$. setosa y $S$. molle mostraron una baja actividad antibacteriana, por el pequeño tamaño de los halos de inhibición a partir de altas concentraciones de los $A E, 40$ y $75 \%$, respectivamente. El AE de $L$. chequen no mostró actividad antimicrobiana sobre S. mutans.

El análisis químico de los $\mathrm{AE}$ reveló la presencia de diversos terpenos y polifenoles con posible actividad antibacteriana, lo que está en relación con estudios previos donde se ha informado que los componentes fenólicos de los $A E$ tales como el eucaliptol, timol, mentona, carvacrol y citral mostraron efecto inhibitorio sobre el crecimiento del $S$. mutans y vienen aplicándose en formulaciones de salud oral.

Al igual que la literatura ${ }^{(5)}$, el $A E$ de $C$. citratus mostró una potente actividad antibacteriana sobre $S$. mutans. Este hallazgo podría explicarse por una mayor actividad biológica de su principal componente "citral", sin embargo, hasta la fecha, el citral y sus derivados no han sido probados en formulaciones de higiene oral para su potencial antimicrobiano. De igual forma el $\mathrm{AE}$ de $P$. elongatum mostró potente actividad antibacteriana contra el S. mutans, probablemente por la presencia del "eucalyptol", un agente antibacteriano ampliamente utilizado en enjuagues bucales, pero también podría resultar de la acción combinada de los diferentes componentes del $\mathrm{AE}$, tal como se ha demostrado previamente en el efecto combinado de los componentes timol, eucalyptol, y salicilato de metilo ${ }^{(1)}$. A diferencia de estos hallazgos los AE de $M$. setosa y $S$. molle mostraron una baja actividad antibacteriana sobre el $S$. mutans, tal como se ha documentado en la literatura ${ }^{(4,5)}$. Una posible explicación es la ausencia de componentes fenólicos con propiedades anticariogénicas en el $\mathrm{AE}$ de $S$. molle, mientras que en el $A E$ de $M$. setosa es posible que la acción combinada de sus componentes pudo inhibir la actividad antibacteriana de los componentes fenólicos mentona y carvacrol, por consiguiente, sus posibles aplicaciones contra el $S$. mutans en el futuro son limitados.

Los hallazgos sugieren que los $A E$ de $C$. citratus y $P$. elongatum podrían emplearse como agentes anticariogénicos para productos de higiene oral, dado su potencial de inhibir el crecimiento del $S$. mutans a partir de bajas concentraciones. Aunque nuestros hallazgos se ven limitados, por la falta de purificación y de ensayos para cada uno de los componentes de estos $\mathrm{AE}$, nuestros hallazgos justifican la importancia de la composición química y la potente actividad antibacteriana encontrada. Por lo tanto, se sugiere realizar más ensayos para cada componente, principalmente del citral, eucaliptol y sus derivados, para su posterior aplicación en formulaciones de higiene oral.

Contribuciones de autoría: EGAA participó en la concepción, diseño, redacción del artículo y aprobación de la versión final. KVAA, BG y VM participaron en la Recolección de datos y análisis e interpretación de datos. MMQF participó en el análisis estadístico.

Fuentes de financiamiento: Autofinanciado.

Conflictos de interés: Los autores declaran no tener conflictos de intereses. 


\section{REFERENCIAS BIBLIOGRÁFICAS}

1. Matute M. Evaluación in vitro del extracto de Piper angustifolium (matico) y la clorhexidina como antisepticos bucales [tesis]. Lima: Universidad Federico Villareal; 2009.

2. Rivadeneira-Cajas D, Álvarez-Velasco P. Aceite esencial de Schinus molle L. (Molle) como potencial antimicrobiano sobre Streptococcus mutans. Estudio in vitro. KIRU. 2015;12(2):8-14.

3. Díaz K, Moromi H. Determinación antibacteriana in vitro de Menthostachysmollis (Muña) frente a bacterias orales de importancia estomatológica. Odontol Sanmarquina. 2005;8(2):3-5.

4. Flores J. Determinación de la actividad antibacteriana "in vitro" del aceite esencial de Luma chequen (Molina) A. Gray "arrayán” frente a Streptococcus mutans. [tesis]. Lima: Universidad Nacional Mayor de San Marcos; 2014.

5. Borja F. Actividad Antibacteriana y Concentración minima inhibitoria del aceite esencial de Cymbopogon citratus frente al Streptococcus mutans in vitro [tesis]. Lima: Universidad Federico Villareal; 2007.

Correspondencia: Elsa Gladys Aguilar Ancori,

Dirección: Urb. Kary Grande A-8 San Sebastian. Cusco. Escuela Profesional de Biología, Universidad Nacional de San Antonio Abad del Cusco. Cusco, Perú.

Correoelectrónico:egaa3@hotmail.com

\section{MEDIDAS DE ASOCIACIÓN EN ESTUDIOS TRANSVERSALES: A PROPÓSITO DEL ESTUDIO "ELEVADA FRECUENCIA DE DISLIPIDEMIA EN PACIENTES INFECTADOS POR VIH EN UN HOSPITAL PÚBLICO PERUANO"}

\author{
ASSOCIATION MEASURES IN CROSS- \\ SECTIONAL STUDIES: CONCERNING THE \\ STUDY "HIGH FREQUENCY OF DYSLIPIDEMIA \\ IN HIV-INFECTED PATIENTS IN A PERUVIAN \\ PUBLIC HOSPITAL"
}

\section{Reyna C. Farfán-García ${ }^{1, a}$, Jorge L. Ulloque ${ }^{1, a}$, Roger V. Araujo-Castillo ${ }^{1, b}$}

Sr. Editor. Hemos leído con gran interés el artículo publicado por Rondan et al. (1) en la revista de la cual usted es editor. Este artículo es de gran importancia ya que evalúa uno de

\footnotetext{
Escuela de Medicina. Universidad Peruana de Ciencias Aplicadas. Lima, Perú

a Estudiante de medicina, ${ }^{\text {b }}$ médico cirujano, internista e infectólogo Recibido: 15/11/2017 Aprobado: 30/11/2017 En línea: 05/04/2018
}

Citar como: Farfán-García RC, Ulloque JL, Araujo-Castillo RV. Medidas de asociación en estudios transversales: a propósito del estudio "Elevada frecuencia de dislipidemia en pacientes infectados por VIH en un hospital público peruano". Rev Peru Med Exp Salud Publica. 2018;35(1):163-4. doi: 10.17843/ rpmesp.2018.351.3611 los principales efectos secundarios producidos por el uso continuo de tratamiento antiretroviral de gran actividad (TARGA), el cual ha sido el pilar para el tratamiento de la infección por el virus de la inmunodeficiencia humana $(\mathrm{VIH})$ durante las últimas décadas. Ante este escenario, se felicita la elección del tema, pues es necesario vigilar a estos pacientes en forma cercana y manejar las posibles complicaciones.

En cuanto al análisis de datos, tanto en la metodología como en las tablas se menciona que la medida de asociación a utilizar será el odds ratio (OR); sin embargo, en la misma metodología se especifica que para el análisis multivariado se usará un modelo lineal generalizado (GLM), familia Poisson link log robusto. Esto difiere con la recomendación usual para modelar OR en forma multivariada, que es usar regresión logística ${ }^{(2)}$. Esta discrepancia en la metodología nos llevó a revisar las tablas de resultados y recalcular los $\mathrm{OR}$, obteniéndose nuevamente una discrepancia entre lo calculado y los resultados presentados. Un examen más minucioso de los resultados nos llevó a encontrar que las cifras presentadas no corresponden a OR sino a razones de prevalencias (RP), incluyendo sus correspondientes intervalos de confianza. No queda claro si también se usaron RP en el modelo multivariado, lo cual concordaría con el enunciado de la metodología de usar regresión de Poisson, ya que este método sería el más adecuado cuando se modelan razones de conteo ${ }^{(3)}$.

Es importante diferenciar entre el uso de OR y RP, y ser cuidadoso con la presentación de los resultados. Si bien los OR son útiles para describir asociaciones en estudios transversales, se recomienda su utilización cuando la prevalencia del desenlace de interés es menor al $10 \%$; de otra forma, se podría sobreestimar la magnitud de la asociación ${ }^{(4)}$. Antes del 2003, era común utilizar OR para modelos multivariados en estudios transversales debido a que no existía otra forma práctica de análisis; hasta que surgió la utilización de modelos log binomiales y modelos lineares generalizados de familia Poisson como alternativas más certeras ${ }^{(5)}$. Desde esa fecha es cada vez más común ver este tipo de análisis en estudios transversales y en estudios de cohorte que utilizan riesgos relativos (RR). De esta manera, hacemos énfasis en la necesidad de ser claros con las medidas de asociación a utilizarse tanto en la metodología como en la presentación de los resultados.

Contribución de autoría: RCFG, JLU y RVAC han participado concepción de la carta al editor, contribuyeron a la elaboración del mismo y aprobaron la versión final.

Fuentes de financiamiento: Autofinanciado.

Conflictos de interés: Ninguno.

\section{REFERENCIAS BIBLIOGRÁFICAS}

1. Rondan PL, Flores-Flores O, Doria NA, Valencia-Mesias G, Chávez-Pérez V, Soria J. Elevada frecuencia de dislipidemia en 